\title{
Soluble Mediators Potentially Involved in Pruritus Associated to Cutaneous T-Cell Lymphomas and Mastocytosis: A Cross-Sectional Study
}

\author{
Coimbra $\mathrm{S}^{1,2 *}$, Mirand $\mathbf{M}^{1,3}$, Abreu $\mathbf{M}^{4,5}$, Lima \\ $M^{4,5,6,7,8 *}$ and Santos-Silva $A^{\mathbf{9 *}}$ \\ ${ }^{1}$ UCIBIO $\backslash$ REQUIMTE, Porto, Portugal \\ ${ }^{2} \mathrm{CESPU}$, Instituto de Investigação e Formação Avançada \\ em Ciências e Tecnologias da Saúde (IINFACTS), Gandra- \\ Paredes, Portugal \\ ${ }^{3}$ Departamento de Ciências Biológicas, Laboratório de \\ Bioquímica, Faculdade de Farmácia, Universidade do \\ Porto (FFUP), Porto, Portugal \\ ${ }^{4}$ Serviço de Hematologia Clínica, Hospital de Santo \\ António (HSA), Centro Hospitalar Universitário do Porto \\ (CHUP), Porto, Portugal \\ 5Instituto de Ciências Biomédicas Abel Salazar, \\ Universidade do Porto (ICBAS/ UP), Porto, Portugal \\ ${ }^{6}$ Consulta Multidisciplinar de Linfomas Cutâneos e \\ Mastocitoses (CMLC), Hospital de Santo António (HSA), \\ Centro Hospitalar Universitário do Porto (CHUP), Porto, \\ Portugal \\ Laboratório de Citometria, Serviço de Hematologia \\ Clínica, Hospital de Santo António (HSA), Centro \\ Hospitalar Universitário do Porto (CHUP), Porto, \\ Portugal \\ ${ }^{8}$ Unidade Multidisciplinar de Investigação Biomédica, \\ Instituto de Ciências Biomédicas Abel Salazar, \\ Universidade do Porto (UMIB/ ICBAS/ UP), Porto, \\ Portugal \\ ${ }^{9}$ UCIBIO $\backslash$ REQUIMTE, Departamento de Ciências \\ Biológicas, Laboratório de Bioquímica, Faculdade de \\ Farmácia, Universidade do Porto (FFUP), Porto, Portugal \\ "Contributed Equally to this Work
}

*Corresponding author: Susana Coimbra, UCIBIO REQUIMTE, Departamento de Ciências Biológicas, Faculdade de Farmácia, Universidade do Porto, R. J orge Viterbo Ferreira 228, 4050-313 Porto, Portugal

Alice Santos-Silva, UCIBIO $\backslash$ REQUIMTE, Departamento de Ciências Biológicas, Faculdade de Farmácia,

Universidade do Porto, R. J orge Viterbo Ferreira 228, 4050-313 Porto, Portugal

Received: April 30, 2021; Accepted: May 27, 2021;

Published: June 03, 2021

\section{Introduction}

Pruritus is a consequence of activation of cutaneous nerve endings by noxious stimuli, namely by inflammatory mediators, neurotransmitters and neuropeptides [1]. Pruritus is a major and distressing symptom, common in inflammatory conditions, such as Cutaneous T Cell Lymphoma (CTCL) and mastocytosis. Initiation and progression of inflammation is associated with various signaling pathways, involving different mediators [2].

CTCL is the most common form of primary cutaneous

\section{Abstract}

Pruritus is a major distressing symptom, common in inflammatory diseases, like Cutaneous T-Cell Lymphoma (CTCL) and mastocytosis. We aimed to study the involvement of some molecules, namely, cytokines, neuromediators, endothelial adhesion molecules and angiogenic factors, in the severity of pruritus associated to CTCL and mastocytosis.

CTCL - Mycosis Fungoides (MF, $n=17$ ) and Sézary syndrome (SS, $n=10)$ and mastocytosis patients $(n=17)$ were evaluated. Interleukin (IL)-8, IL-31, Vascular Endothelial Growth Factor (VEGF), E-selectin, serotonin and C-reactive protein (CRP) levels, were assessed; tryptase was measured in mastocytosis. Pruritus severity was assessed, using a Visual Analogue Scale (VAS).

Compared to controls $(n=29), C T C L$ patients presented higher CRP and IL-31. SS patients had higher IL-31, E-selectin and CRP than MF patients and controls. Itch correlated with IL- 31 and E-selectin, when considering all CTCL patients; in SS, itch correlated with E-selectin. Advanced CTCL stages revealed higher IL-31, E-selectin and CRP than early stages, and controls; itch intensity correlated with IL-31 and E-selectin, in advanced stages. Mastocytosis showed higher serotonin and VEGF, compared to controls, and itch intensity correlated with tryptase.

Data suggest that in mastocytosis, serotonin is an important biomarker and that tryptase levels reflect itch intensity; IL-31 and E-selectin appear to be more important mediators in CTCL and strongly correlated with itch severity. The different involvement of studied mediators, probably due to different immune responses, suggests that different mechanisms underlie these diseases and may lead to different itch mechanisms.

Keywords: Cutaneous T-cell lymphoma; Vascular endothelial growth factor; C-reactive protein
J Blood Disord - Volume 8 Issue 1 - 2021

ISSN 2379-8009 | www.austinpublishing group.com

Coimbra et al. @ All rights are reserved lymphomas. The majority of CTCL are malignant diseases of mature $\mathrm{CD}^{+}$cells, including the variants Mycosis Fungoides (MF) and Sézary Syndrome (SS). Mastocytosis is a heterogeneous group of clinical disorders characterized by accumulation and activation of clonal mast cells in the skin, bone marrow and other organs and/or tissues.

Histamine has been the most studied pruritogen, but antihistamines do not ameliorate pruritus in all pruritus-associated conditions. Non-histaminergic itch is difficult to treat, and several mediators are pointed as possible intervenient in this process [3]. 
Serotonin mediates pruritus that seems to be processed by the same molecular signaling cascade as histamine [4], although independently. Serotonin induced non-histaminergic itch in skin lesions of atopic dermatitis patients [5], and treatment with an antagonist of serotonin receptor did not relieve pruritus induced by histamine in healthy subjects [6].

Cytokines are immunomodulators of the inflammatory response. IL-31 is synthesized by a variety of cells, including lymphocytes, specially Th2 cells, and mast cells [7] that has been proposed as a potential mediator in the pruritus genesis in several diseases. Increased IL-31 has been found in atopic dermatitis [8], mastocytosis [9] and CTCL [10]. IL-31 receptors have been identified in dorsal root ganglion neurons, suggesting that IL-31 may directly activate sensory neurons and cause pruritus, without further interference of other mediators [8].

IL-8 induces neutrophil mobilization, transmigration and degranulation [11]. E-selectin, an adhesion molecule, recruits leukocytes into the site of injury. In mastocytosis, mast cells infiltrated in the bone marrow produce high Vascular Endothelial Growth Factor (VEGF) levels [12]; and, in CTCL, the malignant T cells also release high VEGF levels [13].

The crucial role of the immune response in inflammatory conditions raises the hypothesis that some cytokines and growth factors that are important for inflammatory response may also be involved in the genesis of pruritus. The response to similar therapeutic strategies to treat pruritus is often different for different diseases, strengthening the need to clarify the underlying mechanisms of pruritus and to identify potential mediators involved. We aimed to evaluate, in CTCL and mastocytosis patients, the levels of some mediators that may contribute to pruritus, and to evaluate their relationship with pruritus severity.

\section{Material and Methods}

\section{Subjects}

The study protocol was approved by scientific and ethic committees of Centro Hospitalar Universitário do Porto (CHUP). This study was carried out in accordance with the principles from the Declaration of Helsinki. Subjects were invited to participate and enrolled in the study after informed and written consent, respecting their privacy rights.

Adult patients presenting CTCL $(n=29)$ and mastocytosis $(n=17)$ were included in the study; the control group included 29 healthy volunteers (Table 1).

Diagnosis and classification of CTCL followed the recommendations of the 2018 update of the European Organization for Research and Treatment of Cancer (EORTC) for primary cutaneous lymphomas, and the World Health Organization' classification of tumors of hematopoietic and lymphoid tissues [14]. Lymphoma TNMB (tumor-node-metastasis-blood) staging was established using the ISCL/EORTC revision for Mycosis Fungoides Cooperative Group classification and staging system for CTCL [15]. All CTCL patients had active disease at the time of the study; none of them were receiving chemotherapy at the time of the study. Symptomatic treatment for pruritus included antihistamines $\mathrm{H} 1$ and/ or leukotriene antagonists, such as montelukast.

The diagnosis and classification of mastocytosis were established according to the 2016 updated classification of tumors of hematopoietic and lymphoid tissues [16]. All mastocytosis patients were studied while following-up the disease and they were only under symptomatic treatment, which included antihistamines $\mathrm{H} 1$ and $\mathrm{H} 2$, leukotriene antagonists, such as montelukast, and/or mast cell stabilizers, such as ketotifen and sodium chromoglycate.

The intensity of pruritus was assessed by using a Visual Analogue Scale (VAS), ranging from 0 (absence of itch) to 10 (the severest itch ever felt by the subject).

\section{Analytical assays}

Blood was collected and processed within 2 hours, in order to obtain serum; serum aliquots were immediately stored at $-80^{\circ} \mathrm{C}$ until assayed.

IL-8, IL-31 (BioLegend, California, USA), VEGF, E-selectin, (eBioscience, California, USA), C-reactive protein (CRP; R\&D Systems, Minneapolis, USA) and serotonin (Enzo Life Sciences, New York, USA) were quantified using Enzyme-Linked Immunosorbent Assays (ELISA).

In mastocytosis, tryptase levels were evaluated by FluoroImmuno-Enzymatic Assay (ImmunoCap, ThermoFisher Scientific, Massachusetts, USA).

\section{Statistical analysis}

Statistical analysis was performed using the Statistical Package for Social Sciences (SPSS, version 22.0, Chicago, IL, USA) for Windows. Data is presented as median levels [interquartile range, IQR]. To evaluate differences between groups, the Kruskal-Wallis $\mathrm{H}$ test was used and, when statistical significance was achieved, single comparisons (two groups) were made by using the Mann-Whitney $\mathrm{U}$ test. Spearman's rank correlation coefficient was used to evaluate relationships between sets of data. A P value $<0.05$ was considered statistically significant.

\section{Results}

Considering CTCL patients, $58.5 \%$ were diagnosed with MF and $34.5 \%$ with SS (Table 1). Using the TMNB staging, 10 patients were at stage Ia, 9 at stage Ib, 1 at stage IIb, 4 at stage IIIb, and 5 at stage IVa; totalizing $69 \%$ patients with early and $31 \%$ patients with advanced disease. In all cases, the lymphoma originated from $\mathrm{CD} 4^{+} \mathrm{T}$ cells.

The median itch intensity in CTCL (Table 2) was of 1.0 (IQR: [0.0-9.0]); the SS patients, presented higher values than MF patients. CTCL patients with advanced disease presented higher itch intensity.

Mastocytosis patients had predominantly systemic mastocytosis (SM; 88.2\%); and all patients showed maculopapular skin lesions (Urticaria Pigmentosa) (Table 1). The KIT D816V mutation was positive in 13 cases. Tryptase levels were increased in 13 patients, being higher than $20 \mathrm{ng} / \mathrm{mL}$ in 8 cases (minor criteria for the diagnosis of SM), and exceeding $200 \mathrm{ng} / \mathrm{mL}$ in two patients (both with smoldering SM).

The median itch intensity in mastocytosis patients was of 1.0 (IQR: [0.0-1.5]). 
Table 1: Demographic and clinical data for controls and for Cutaneous T-Cell Lymphoma (CTCL) and mastocytosis patients.

\begin{tabular}{|c|c|}
\hline Controls $(n=29)$ & \\
\hline Age, years & 58 [57-62] \\
\hline Gender, males/females & $17(58.6 \%) / 12(41.4 \%)$ \\
\hline \multicolumn{2}{|l|}{ CTCL (n=29) } \\
\hline Age, years & 'b6 [55-74] \\
\hline Gender, males/females & $15(51.7 \%) / 14(48.3 \%)$ \\
\hline \multicolumn{2}{|l|}{ Disease classification } \\
\hline Mycosis Fungoides & $17(58.5 \%)$ \\
\hline Classical MF & $15(51.7 \%)$ \\
\hline MF variants & $2(6.9 \%)$ \\
\hline Sézary Syndrome & $10(34.5 \%)$ \\
\hline Primary cutaneous CD30+ ALCL & $1(3.4 \%)$ \\
\hline CTCL, NOS & $1(3.4 \%)$ \\
\hline \multicolumn{2}{|l|}{ Disease stage } \\
\hline Early disease (stage I + II) & $20(69.0 \%)$ \\
\hline Advanced disease (stages III + IV) & $9(31.0 \%)$ \\
\hline \multicolumn{2}{|l|}{ Mastocytosis $(n=17)$} \\
\hline Age, years & bb $43[36-58]^{c c}$ \\
\hline Gender, males & $5(29.4 \%) / 12(70.6 \%)$ \\
\hline \multicolumn{2}{|l|}{ Disease classification } \\
\hline Cutaneous Mastocytosis & $2(11.8 \%)$ \\
\hline Systemic Mastocytosis & $15(88.2 \%)$ \\
\hline Indolent SM & $11(64.7 \%)$ \\
\hline Smoldering SM & $2(11.8 \%)$ \\
\hline Aggressive SM & $0(0.0 \%)$ \\
\hline Mast cell leukemia & $0(0.0 \%)$ \\
\hline $\mathrm{SM}-\mathrm{AHN}$ a & $2(11.8 \%)$ \\
\hline \multicolumn{2}{|c|}{ KIT D816V mutation in the peripheral blood aa, aaa } \\
\hline Positive & $13(76.5 \%)$ \\
\hline Negative & $4(23.5 \%)$ \\
\hline Serum tryptase levels, ng/mL & $15.9[9.1-42.6]$ \\
\hline Normal $(\leq 11.4)$ & $4(23.5 \%)$ \\
\hline Increased (>11.4) & $13(76.5 \%)$ \\
\hline Increased (>20) ааа & $8(47.1 \%)$ \\
\hline
\end{tabular}

ALCL: Anaplastic Large Cell Lymphoma; MF: Mycosis Fungoides; NOS Not Otherwise Specified; SM: Systemic Mastocytosis; SM-AHN: Systemic Mastocytosis Associated to other non-mast cell Hematological Neoplasm.

Data is presented as median values [interquartile range, IQR] or as absolute and relative frequencies, expressed as percentages (\%).

aOne patient had Indolent SM associated with Essential Thrombocytemia and another patient had ISM associated with Refractory Anemia with Ring

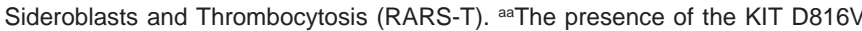
mutation was evaluated in DNA extracted from total peripheral blood cells, using an Allele-Specific Quantitative Oligonucleotide Polymerase Chain Reaction (ASO-qPCR) (sensitivity: 0.01\%). aaaMinor criteria for the diagnosis of SM.

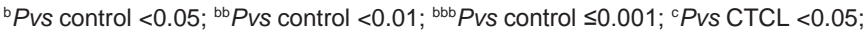
${ }^{c c} P$ Vs CTCL<0.01; ${ }^{c c c} P$ Vs CTCL $\leq 0.001$.

Patients and controls were matched for gender; CTCL patients were older than controls, while mastocytosis patients were younger (Table 1).
As compared to controls (Table 2), mastocytosis patients showed higher serotonin and VEGF and a trend towards higher CRP $(\mathrm{P}=0.092)$; while CTCL patients presented higher CRP and IL-31, as well as a trend towards higher $\mathrm{E}$-selectin $(\mathrm{P}=0.077)$ and serotonin $(\mathrm{P}=0.065)$.

Considering CTCL, SS patients (Table 2) presented higher IL31, E-selectin and CRP than MF patients and controls; MF patients showed a trend towards higher serotonin, when compared to controls $(\mathrm{P}=0.074)$.

Patients at advanced CTCL stages (stages III and IV) presented higher IL-31, E-selectin and CRP, than patients at early stages of the disease and controls (Table 2).

In mastocytosis, the itch score correlated with tryptase (Figure 1); in CTCL, itch score correlated with age $(\mathrm{rS}=0.511 ; \mathrm{P}=0.005)$, IL-31 and E-selectin; in CTCL patients with advanced disease, itch intensity correlated with IL-31 and E-selectin; SS patients presented also a positive correlation between, itch score and E-selectin (Figure 1).

In mastocytosis, serotonin correlated inversely with IL-8 (rS=$0.645 ; \mathrm{P}=0.005)$ and with tryptase $(\mathrm{rS}=-0.624 ; \mathrm{P}=0.007)$.

In CTCL, IL-31 correlated with age ( $\mathrm{rS}=0.511 ; \mathrm{P}=0.005)$, IL-8 ( $\mathrm{rS}=0.395 ; \mathrm{P}=0.037), \mathrm{CRP} \quad(\mathrm{rS}=0.599 ; \mathrm{P}=0.001)$ and E-selectin $(\mathrm{rS}=0.520 ; \mathrm{P}=0.005)$; CRP correlated with IL-8 ( $\mathrm{rS}=0.493 ; \mathrm{P}=0.007)$, E-selectin $(r S=0.613 ; \mathrm{P}<0.001)$ and VEGF $(r S=0.371 ; \mathrm{P}=0.047)$.

In MF and early stages of CTCL, IL-31 correlated with IL-8 $(\mathrm{rS}=0.539, \mathrm{P}=0.026 ; \mathrm{rS}=0.534, \mathrm{P}=0.015$; respectively) and with $\mathrm{CRP}$ $(\mathrm{rS}=0.545, \mathrm{P}=0.024 ; \mathrm{rS}=0.504, \mathrm{P}=0.023$; respectively); IL-8 correlated with CRP ( $\mathrm{rS}=0.555 ; \mathrm{P}=0.021 ; \mathrm{rS}=0.484, \mathrm{P}=0.031$; respectively) and E-selectin ( $\mathrm{rS}=0.492, \mathrm{P}=0.045 ; \mathrm{rS}=0.493, \mathrm{P}=0.027$; respectively); MF patients showed also a correlation between IL-31 and VEGF $(\mathrm{rS}=0.544 ; \mathrm{P}=0.024)$. In CTCL patients with advanced disease, E-selectin correlated with CRP $(\mathrm{rS}=0.900, \mathrm{P}=0.001)$; and VEGF correlated with IL-8 $(\mathrm{rS}=0.828, \mathrm{P}=0.006)$. In SS patients, CRP correlated with E-selectin $(\mathrm{rS}=0.891 ; \mathrm{P}=0.001)$; and IL-8 correlated with VEGF ( $\mathrm{rS}=0.863 ; \mathrm{P}=0.001)$.

\section{Discussion}

CTCL and mastocytosis are associated with inflammation, showed by the enhanced CRP levels. Our data suggest an important role for E-selectin, CRP and IL-31 in CTCL. These patients presented higher IL-31 and CRP, as compared with controls; IL-31 correlated with E-selectin and CRP, and these last two were also correlated; E-selectin and IL-31 seem to reflect the severity of inflammation in these patients. Both IL-31 and E-selectin were correlated with itch intensity, suggesting that, in CTCL, these two biomarkers are important for itch development and worsening. In advanced stages of CTCL and in SS patients, presenting the highest pruritus, these correlations were even more significant.

The SS and the CTCL patients at advanced stages of the disease showed higher IL-31, E-selectin and CRP, compared to MF patients and to CTCL patients at early stages, and to controls; both groups showed a correlation between CRP and E-selectin. It seems that the inflammatory process is more pronounced in SS and in late CTCL stages than in MF and early stage CTCL and contributes for development/ 
Table 2: Analytical data and pruritus intensity for controls, mastocytosis patients and for subjects with Cutaneous T-Cell Lymphoma (CTCL), according to the variants Mycosis Fungoides (MF) and Sézary Syndrome (SS) and according to stage of disease.

\begin{tabular}{|c|c|c|c|c|c|c|c|}
\hline Solublemediators & $\begin{array}{l}\text { Controls } \\
(n=29)\end{array}$ & $\begin{array}{l}\text { Mastocytosis } \\
\quad(n=17)\end{array}$ & CTCL $(n=29)$ & MF $(n=17)$ & SS $(n=10)$ & $\begin{array}{l}\text { Early stages }(I+I I) \\
\qquad(\mathrm{n}=20)\end{array}$ & $\begin{array}{l}\text { Late stages }(I I I+I V) \\
\qquad(n=9)\end{array}$ \\
\hline IL-8 (pg/mL) & $9.3[5.4-20.6]$ & $8.0[4.8-36.8]$ & $10.2[4.8-26.6]$ & $11.1[4.8-26.6]$ & $13.0[4.9-30.1]$ & $9.8[4.8-26.3]$ & $15.8[4.6-37.8]$ \\
\hline IL-31 (pg/mL) & $7.8[2.2-15.2]$ & $6.8[2.3-30.4]$ & a 23.1 [6.1-29.2] & $11.4[3.7-26.6]$ & $\underset{\text { bb }}{\text { aaa }} 28.3$ [25.1-139.9] & $10.9[3.7-26.0]$ & aaa $28.3[25.1-53.1]^{\mathrm{c}}$ \\
\hline Serotonin (ng/mL) & 165 [101-208] & aa 240 [155-261] & 182 [161-240] & 186 [161-243] & $240[155-261]$ & 181 [161-239] & 201 [129-244] \\
\hline E-selectin (ng/mL) & $39.1[28.9-43.7]$ & 36.1 [28.6-53.7] & $44.0[31.2-84.3]$ & $35.3[25.9-54.7]$ & aa $84.3[43.4-156.4]^{\text {b }}$ & $35.0[29.4-48.9]$ & aаa $93.5[43.7-167.6]^{\mathrm{cc}}$ \\
\hline VEGF (pg/mL) & 549 [366-678] & a 849 [441-1243] & 653 [429-879] & 749 [429-822] & 687 [330-1396] & $640[507-774]$ & $720[323-1464]$ \\
\hline CRP $(\mu \mathrm{g} / \mathrm{mL})$ & $0.71[0.33-1.02]$ & 1.19 [0.51-5.48] & a $1.35[0.51-5.48]$ & $0.78[0.23-2.71]$ & aaa $3.86[1.04-8.47]^{b}$ & $0.90[0.35-2.50]$ & aaa $6.14[1.21-9.79]^{c}$ \\
\hline Pruritus (VAS) & & $1.0[0.0-1.5]$ & $1.0[0.0-9.0]$ & $0.0[0.0-1.5]$ & $9.0[7.3-10.0]^{\mathrm{bb}}$ & $0.0[0.0-1.0]$ & $10.0[8.0-10.0]^{\mathrm{ccc}}$ \\
\hline
\end{tabular}

IL: Interleukin; CRP: C-Reactive Protein; VAS: Visual Analogue Scale; VEGF: Vascular Endothelial Growth Factor.

Data is presented as median levels [interquartile range, IQR].

${ }^{\text {aPV }}$ control $<0.05$; ${ }^{\text {aa }}$ PVs control $<0.01$; ${ }^{\text {aaa }}$ PVs control $\leq 0.001$.

${ }^{\mathrm{b} P}$ V $\mathrm{MF}<0.05$; ${ }^{\mathrm{b}} \mathrm{P}$ V $\mathrm{MF}<0.01$.

${ }^{c} P V S$ early stages $<0.05$; ${ }^{c c} P V S$ early stages $<0.01$; ${ }^{c c} P V s$ early stages $<0.001$.
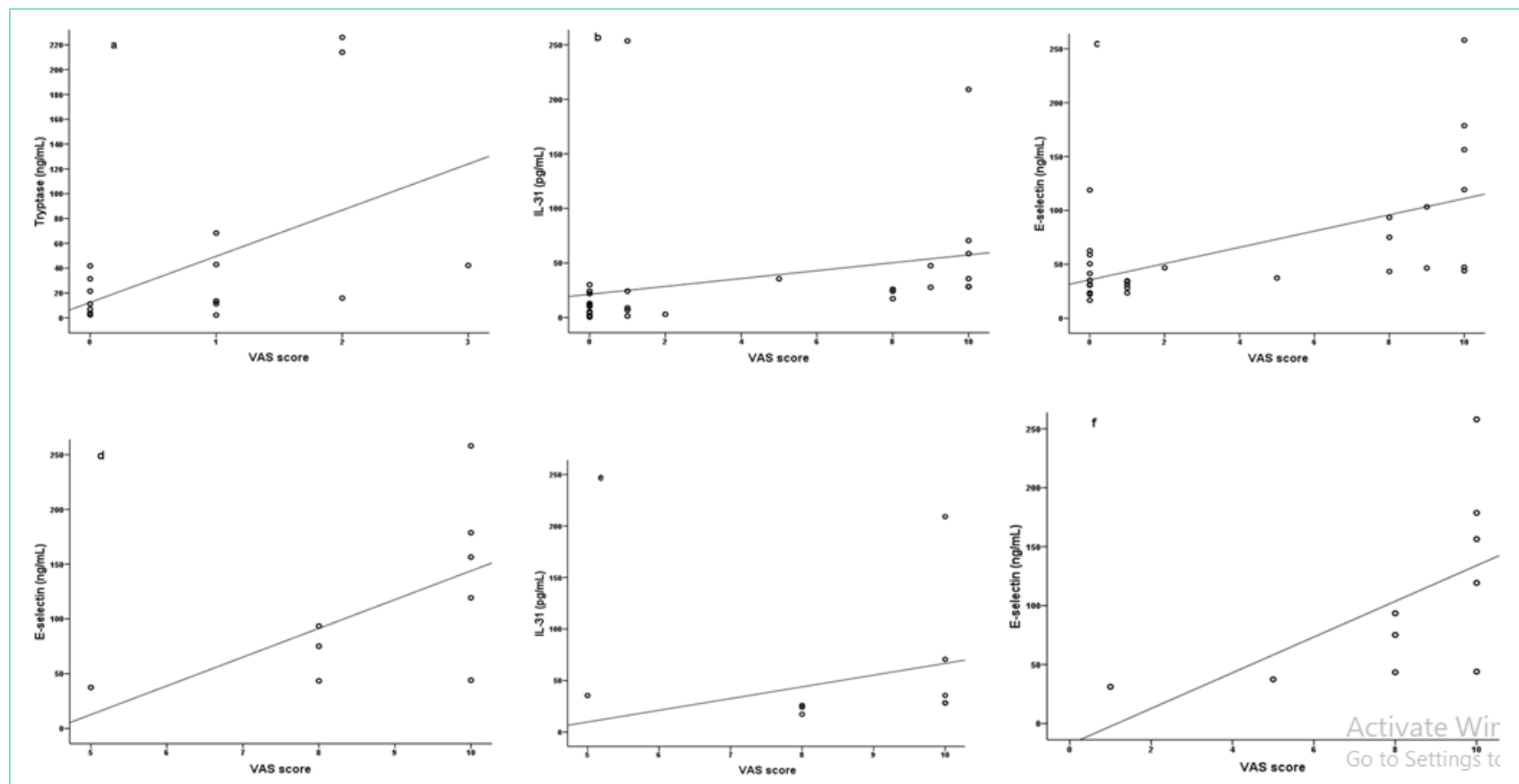

Figure 1: Correlations found between itch, assessed through VAS (Visual Analogue Scale), and tryptase ( $r S=0.515 ; P=0.034)$, in mastocytosis (a); IL-31 (rS = 0.675; $\mathrm{P}<0.001)$ and E-selectin ( $\mathrm{rS}=0.564 ; \mathrm{P}=0.001)$, in all CTCL patients (b and c, respectively); E-selectin ( $\mathrm{rS}=0.745 ; \mathrm{P}=0.021)$ and IL-31 ( $\mathrm{rS}=0.850 ; \mathrm{P}=$ 0.007 ) in advanced CTCL stages ( $d$ and e, respectively); and E-selectin ( $r S=0.884 ; P=0.004$ ) in Sézary Syndrome patients $(f)$.

worsening of pruritus. IL-31 is produced by the malignant T cell clone [17], and skin homing of malignant cells from SS patients seems to be mediated, in a dependent manner, by E-selectin [18]. Our data are in accordance with these findings, suggesting that IL-31 and E-selectin are important players in CTCL mechanisms. The high IL-31 levels are likely to result from an overwhelming production by $\mathrm{T}$ cell malignant clone, and the elevated E-selectin may contribute to the development of erythroderma, observed in SS patients.

In advanced disease and in SS, itch intensity correlated with E-selectin, and, in advanced stages, itch was also correlated with IL31. Previous studies in psoriatic lesional skin indicated that E-selectin is one of the possible mediators involved in pruritus in psoriasis, along with neurogenic factors, activated mast cells and some cytokines [19].
In atopic dermatitis, E-selectin was found to correlate with the clinical index SCORAD, a tool that evaluates the area affected, intensity of some signs, and subjective symptoms, namely itch [20]. E-selectin mediates homing of $\mathrm{T}$ lymphocytes from SS patients in rat skin [18], suggesting an important role for E-selectin in SS mechanism. The association found between E-selectin and itch score, suggest that E-selectin is important for itch pathway in CTCL, especially in SS and late stage patients, conditions that were associated with an enhancement in inflammation, probably resulting from a complex interplay between E-selectin, inflammation and T-lymphocytes. Disruption of IL-31 production by malignant $\mathrm{T}$ lymphocytes successfully abolished pruritus in SS patients [21], suggesting that IL-31 may also be important for the pruritus mechanism in CTCL. We found a positive correlation between itch and IL-31 that was even 
higher in advanced disease, further strengthening the role proposed for this cytokine in pruritus development/worsening in CTCL. Our data show that IL-31, E-selectin and CRP are especially enhanced in advanced stages of CTCL and in SS patients, suggesting a complex pruritus mechanism, involving several mediators.

A correlation of age with IL-31 and with itch score was observed, which was not found for controls and mastocytosis patients. It seems that older CTCL patients are more prone to enhancement of pruritus and of IL-31 levels, probably as a consequence of immune system compromise with aging.

Mastocytosis is characterized by an accumulation of clonal mast cells in different organs and tissues. Tryptase, a serine proteinases abundant in the secretory granules of mast cells, was reported to induce pruritus in mice, in a mechanism involving ProteaseActivated Receptor-2 (PAR-2) [22]. In atopic dermatitis, both in a mice model and in human skin, tryptase and PAR-2 were reported to stimulate itch $[23,24]$. According to our data, in mastocytosis, tryptase levels are closely related with pruritus intensity. Tryptase may, actually, provide a marker of mast cell burden and activation and, furthermore, a biomarker of itch severity.

Human mast cells are also able to produce and release serotonin $[25,26]$. In our study, and in agreement with others, mastocytosis patients presented higher serotonin levels [25]. In mastocytosis, serotonin levels are distributed in a bimodal manner and are not correlated with tryptase levels [26]. In our study, tryptase levels correlated inversely with serotonin. According to the study previously referred [26], patients with low serotonin levels were more likely to present neurological and gastrointestinal disturbances. Mast cell proliferation and activation is altered in mastocytosis, since the neoplastic cells present activating mutation (usually D816V) in KIT, the gene codifying for a transmembrane receptor for stem cell factor (CD117) that is crucial for mast cell growth and differentiation [27] As pointed by the authors [26], it is possible that storage and release of serotonin in altered mast cells, or the degradation of serotonin by mast cells products, contributes to abnormal serotonin levels, explaining its little contribution to the clinical symptoms observed, and, in the present study, to the negative correlation found with tryptase. We can also speculate that an association between low serotonin levels and more severe forms of mastocytosis may exist, explaining the correlation found.

Mast cells also secrete growth factors, as VEGF, one of the most important inducers of angiogenesis. Apparently, substance P induces the production of VEGF by mast cells [28]. We found enhanced VEGF in mastocytosis. SM has been associated with increased bone marrow angiogenesis and with VEGF expression in mast cells infiltrates [12], suggesting an important role for VEGF in the etiopathogenic mechanism of mastocytosis.

The existence of higher IL-31 and E-selectin in CTCL, especially in SS and advanced stages, and of high serotonin in mastocytosis patients, may be related to the type of immune response involved in these conditions. Early CTCL stages present a dominance of the Th1 profile; while in advanced stages occur a switch to a Th2 profile. In early phases of MF, a T-helper (Th)1-biased response predominates, whereas a Th2-type response is found in advanced MF cases; SS has a Th2-type profile [29]; in mastocytosis, the Th2 pathway seems to have an important role [17]. It is possible that the involvement of different pathways in these disorders explain the different levels of the currently studied biomarkers. In CTCL, the differences found between early and advanced stages and between MF and SS, and the correlations found for itch in the advanced and SS groups, may result from the switch from Th1 to Th2.The increase in inflammation, observed in late MF and in SS patients seems to be, actually, an important factor for the differences found, when comparing early versus advanced stages of CTCL, and MF versus SS patients.

Our data suggest that serotonin is an important biomarker in mastocytosis, and that tryptase levels reflect itch intensity in this condition, whereas IL-31 and E-selectin appear to be important mediators in CTCL, acting as biomarkers of disease progression and development/worsening of itch. The involvement of different mediators in these two inflammatory conditions, which are probably due to different immune responses, not only suggests that different mechanisms underlie the genesis of these diseases, and may lead for different itch mechanisms, but also explains the different therapeutic responses to currently available treatments for itch, highlighting the work that is still needed to optimize the treatment of pruritus.

\section{Acknowledgment}

This work was supported by the Applied Molecular Biosciences Unit (UCIBIO), financed by national funds from FCT (UIDP/04378/2020 and UIDB/04378/2020); by the North Portugal Regional Coordination and Development Commission (CCDR-N)/ NORTE2020/Portugal 2020 (Norte-01-0145-FEDER-000024); by CESPU/IINFACTS (PI3AC_2016); by CHP, ICBAS/UP and UMIB/ ICBAS/UP.

\section{References}

1. Wong LS, Wu T, Lee CH. Inflammatory and Noninflammatory Itch: Implications in Pathophysiology-Directed Treatments. Int J Mol Sci. 2017; 18: 1485.

2. Yeung YT, Aziz F, Guerrero-Castilla A, Arguelles S. Signaling Pathways in Inflammation and Anti-inflammatory Therapies. Curr Pharm Des. 2018; 24: 1449-1484.

3. Grundmann-Kollmann M, Ludwig R, Zollner TM, Ochsendorf F, Thaci D, Boehncke WH, et al. Narrowband UVB and cream psoralen-UVA combination therapy for plaque-type psoriasis. J Am Acad Dermatol. 2004; 50: 734-739.

4. Kremer AE, Feramisco J, Reeh PW, Beuers U, Oude Elferink RP. Receptors, cells and circuits involved in pruritus of systemic disorders. Biochim Biophys Acta. 2014; 1842: 869-892.

5. Hosogi M, Schmelz M, Miyachi $Y$, Ikoma A. Bradykinin is a potent pruritogen in atopic dermatitis: a switch from pain to itch. Pain. 2006; 126: 16-23.

6. Weisshaar E, Ziethen B, Gollnick H. Can a serotonin type 3 (5-HT3) receptor antagonist reduce experimentally-induced itch? Inflamm Res. 1997; 46: 412 416.

7. Cornelissen C, Luscher-Firzlaff J, Baron JM, Luscher B. Signaling by IL-31 and functional consequences. Eur J Cell Biol. 2012; 91: 552-566.

8. Sonkoly E, Muller A, Lauerma Al, Pivarcsi A, Soto H, Kemeny L, et al. IL-31 a new link between $\mathrm{T}$ cells and pruritus in atopic skin inflammation. J Allergy Clin Immunol. 2006; 117: 411-417.

9. Stratis A, Pasparakis M, Rupec RA, Markur D, Hartmann K, ScharffetterKochanek K, et al. Pathogenic role for skin macrophages in a mouse mode of keratinocyte-induced psoriasis-like skin inflammation. J Clin Invest. 2006; 116: 2094-2104.

10. Ohmatsu H, Sugaya M, Suga H, Morimura S, Miyagaki T, Kai H, et al. Serum IL-31 levels are increased in patients with cutaneous T-cell lymphoma. Acta 
DermVenereol. 2012; 92: 282-283.

11. Mukaida N. Interleukin-8: an expanding universe beyond neutrophil chemotaxis and activation. Int J Hematol. 2000; 72: 391-398.

12. Wimazal F, Jordan JH, Sperr WR, Chott A, Dabbass S, Lechner K, et al. Increased angiogenesis in the bone marrow of patients with systemic mastocytosis. The Am J Pathol. 2002; 160: 1639-1645.

13. Eriksen KW, Lovato P, Skov L, Krejsgaard T, Kaltoft K, Geisler C, et al. Increased sensitivity to interferon-alpha in psoriatic T cells. J Invest Dermatol. 2005; 125: 936-944.

14. Willemze R, Cerroni L, Kempf W, Berti E, Facchetti F, Swerdlow SH, et al The 2018 update of the WHO-EORTC classification for primary cutaneous lymphomas. Blood. 2019; 133: 1703-1714.

15. Olsen AO, Grjibovski A, Magnus P, Tambs K, Harris JR. Psoriasis in Norway as observed in a population-based Norwegian twin panel. $\mathrm{Br} \mathrm{J}$ Dermatol. 2005; 153: 346-351.

16. Valent P, Akin C, Metcalfe DD. Mastocytosis: 2016 updated WHO classification and novel emerging treatment concepts. Blood. 2017; 129: 1420-1427.

17. Singer EM, Shin DB, Nattkemper LA, Benoit BM, Klein RS, Didigu CA, et al. IL-31 is produced by the malignant T-cell population in cutaneous T-Cell lymphoma and correlates with CTCL pruritus. J Invest Dermatol. 2013; 133: 2783-2785.

18. Hoeller C, Richardson SK, Ng LG, Valero T, Wysocka M, Rook AH, et al. In vivo imaging of cutaneous T-cell lymphoma migration to the skin. Cancer Res. 2009; 69: 2704-2708

19. Nakamura M, Toyoda M, Morohashi M. Pruritogenic mediators in psoriasis vulgaris: comparative evaluation of itch-associated cutaneous factors. $\mathrm{Br} \mathrm{J}$ Dermatol. 2003; 149: 718-730.

20. Gutgesell C, Heise S, Seubert A, Stichtenoth DO, Frolich JC, Neumann C Comparison of different activity parameters in atopic dermatitis: correlation with clinical scores. Br J Dermatol. 2002; 147: 914-919.
21. Cedeno-Laurent F, Singer EM, Wysocka M, Benoit BM, Vittorio CC, Kim EJ, et al. Improved pruritus correlates with lower levels of IL-31 in CTCL patients under different therapeutic modalities. Clin Immunol. 2015; 158: 1-7.

22. Ui H, Andoh T, Lee JB, Nojima H, Kuraishi Y. Potent pruritogenic action of tryptase mediated by PAR-2 receptor and its involvement in anti-pruritic effect of nafamostatmesilate in mice. Eur J Pharmacol. 2006; 530: 172-178.

23. Zhu Y, Pan WH, Wang XR, Liu Y, Chen M, Xu XG, et al. Tryptase and protease-activated receptor-2 stimulate scratching behavior in a murine model of ovalbumin-induced atopic-like dermatitis. Int Immunopharmacol. 2015; 28: 507-512.

24. Steinhoff M, Neisius U, Ikoma A, Fartasch M, Heyer G, Skov PS, et al. Proteinase-activated receptor-2 mediates itch: a novel pathway for pruritus in human skin. J Neurosci. 2003; 23: 6176-6180.

25. Kushnir-Sukhov NM, Brown JM, Wu Y, Kirshenbaum A, Metcalfe DD. Human mast cells are capable of serotonin synthesis and release. J Allergy Clin Immunol. 2007; 119: 498-499.

26. Kushnir-Sukhov NM, Brittain E, Scott L, Metcalfe DD. Clinical correlates of blood serotonin levels in patients with mastocytosis. Eur J Clin Invest. 2008; 38: 953-958.

27. Metcalfe DD. Regulation of normal and neoplastic human mast cell development in mastocytosis. Trans Am Clin Climatol Assoc. 2005; 116: 185204.

28. Shaik-Dasthagirisaheb YB, Varvara G, Murmura G, Saggini A, Potalivo G, Caraffa A, et al. Vascular Endothelial Growth Factor (VEGF), mast cells and inflammation. Int J Immunopathol Pharmacol. 2013; 26: 327-335.

29. Papadavid E, Economidou J, Psarra A, Kapsimali V, Mantzana V, Antoniou $C$, et al. The relevance of peripheral blood T-helper 1 and 2 cytokine pattern in the evaluation of patients with mycosis fungoides and Sezary syndrome. $\mathrm{Br}$ J of Dermatol. 2003; 148: 709-718. 